\title{
Levantamento da Infestação de Plantas Aquáticas em Porto Primavera antes do Enchimento Final do Reservatório ${ }^{1}$
}

\author{
Aquatic Plant Infestation Assessment in Porto Primavera Reservoir Before Final Filling
}

MARTINS, D. ${ }^{2}$, PITELLI, R.A. ${ }^{3}$, TOMAZELLA, M.S. ${ }^{4}$, TANAKA, R.H..${ }^{5}$ e RODRIGUES, A.C.P. ${ }^{6}$

\begin{abstract}
RESUMO - O objetivo deste trabalho foi identificar as plantas aquáticas e os niveis de infestação de cada espécie em Porto Primavera antes do enchimento final do reservatório. Foram avaliados todos os focos de vegetação aquática no reservatório (72 pontos), sendo os pontos demarcados com um aparelho de GPS. As espécies foram identificadas e estimouse visualmente (tamanho da área) a distribuição proporcional das plantas no foco de infestação. Após a identificação, foram encontradas 18 espécies de plantas aquáticas vegetando no reservatório, das quais foram determinadas a frequência de espécie de planta aquática e a distribuição dentro do sistema de geração de energia. As espécies encontradas no reservatório foram: Eichhornia crassipes, Eichhornia azurea, Pistia stratiotes, Paspalum repens, Cyperus brevifolius, Paspalum conspersum, Echinochloa polystachya, Egeria densa, Egeria najas, Polygonum hidropiperoides, Polygonum lapathifolium, Alternanthera philoxeroides, Eleocharis sellowiana, Nymphaea ampla, Pontederia cordata, Salvinia auriculata, Salvinia rotundifolia e Typha angustifolia. As maiores frequências relativas foram observadas em: E. azurea $(36,11 \%)$, E. crassipes $(16,67 \%)$, P. stratiotes $(13,89 \%)$, S. auriculata $(13,89 \%)$, C. brevifolius $(11,11 \%)$ e P. lapathifolium $(6,94 \%)$.
\end{abstract}

Palavras-chave: planta aquática, reservatório, planta daninha.

\begin{abstract}
The objective of this work was to identify the aquatic plants and their infestation levels in Porto Primavera Reservoir in the state of São Paulo, Brazil, before the reservoir was filled. The aquatic vegetation in the dam (72 sites) were evaluated and marked with GPS equipment. The species were identified and a visual estimate (site size) of the proportional distribution of the plants in the infestation foci was carried out. After plant identification, eighteen macrophyte species were found in the dam and their frequency and distribution were determined as follows: Eichhornia crassipes, Eichhornia azurea, Pistia stratiotes, Paspalum repens, Cyperus brevifolius, Paspalum conspersum, Echinochloa polystachya, Egeria densa, Egeria najas, Polygonum hidropiperoides, Polygonum lapathifolium, Alternanthera philoxeroides, Eleocharis sellowiana, Nymphaea ampla, Pontederia cordata, Salvinia auriculata, Salvinia rotundifolia, Typha angustifolia. The highest relative frequencies were observed in: E. azurea (36.11\%), E. crassipes (16.67\%), P. stratiotes (13.89\%), S. auriculata (13.89\%), C. brevifolius (11.11\%), and P. lapathifolium (6.94\%).
\end{abstract}

Keywords: aquatic plant, reservoir, weed.

1 Recebido para publicação em 2.10.2008 e na forma revisada em 11.12.2009.

2 Prof. Livre Docente, Depto. de Produção Vegetal, FCA/UNESP, Caixa Postal 237, 18603-970 Botucatu-SP, $<$ dmartins@fca.unesp.br>; ${ }^{3}$ Prof. Titular, FCAV/UNESP, Via de Acesso Prof. Paulo Donato Castellane, s/n, 14884-900 Jaboticabal-SP, <rapitelli@ecosafe.agr.br>; ${ }^{4}$ Eng $^{0}-$ Agr $^{\circ}$., Dr., Defesa Agropecuária, Av. Brasil 2340, 1303-000 Campinas-SP, <tomazella@cda.gov.br>; ${ }^{5}$ Engô-Agro $^{-}$, M.Sc., CPFL, Rodovia Campinas Mogi-Mirim km 2,5, < robsontanaka@cpfl.com.br> ${ }^{6}$ Aluna de pós-graduação do Depto. de Produção Vegetal, FCA/UNESP, Caixa Postal 237, 18603-970 Botucatu-SP, <andreia@fca.unesp.br>. 


\section{INTRODUÇÃO}

As plantas aquáticas são componentes importantes em corpos hídricos, pois desempenham papel fundamental no estoque de energia e carbono nas bases das pirâmides alimentares, promovem habitats adequados para muitos organismos e servem de suporte à ovoposição e ao refúgio de peixes e outros animais (Neves et al., 2002). Contudo, a vegetação aquática passa a ser considerada como daninha quando seu crescimento acentuado causa problemas para a utilização dos ecossistemas, como navegação, pesca, esportes náuticos, entre outros, surgindo a necessidade de aplicação de métodos de controle ou manejo (Pitelli, 1998; Martins et al., 2008).

De acordo com Branco (1986), existem várias espécies macrófitas com potencial para causar prejuízos em ecossistemas aquáticos. Os aguapés (gênero Eichhornia), por exemplo, têm sido motivo de grande preocupação nas represas da companhia Light. As plantas dessa espécie tendem a cobrir a superfície das represas, com perigo de serem absorvidas ou entupirem as grades de proteção dos sistemas de turbinas geradoras de energia elétrica.

Em contraste com os prejuízos já constatados desse tipo de vegetação, destaca-se a ausência, no Brasil, de legislação específica envolvendo o monitoramento e controle de plantas aquáticas. Em outros países, com destaque para os EUA, a situação é completamente diferente. Em alguns casos, como no estado da Flórida, o controle de plantas aquáticas é considerado uma questão de segurança pública, tendo sido criado um imposto que incide sobre a comercialização de combustiveis especificamente para financiar pesquisas e ações voltadas para o monitoramento e controle das plantas aquáticas (Velini, 1998).

Apesar de haver técnicas avançadas para determinar a vegetação aquática de uma região, como o uso do sensoriamento remoto orbital (Martyn et al., 1986; Marshall \& Lee, 1994; Galo et al., 2002), acredita-se que, tratando de identificação de espécie, técnicas mais simples, como a visualização e identificação dos indivíduos no habitat natural, podem ser mais eficientes, devido à maior precisão na verificação das características inerentes a cada espécie durante o levantamento (Martins et al., 2008).

O levantamento de infestação por plantas aquáticas faz-se necessário, pois permite verificar o grau de infestação das diferentes espécies e a distribuição destas nos corpos d'água estudados. A partir desses dados, decisões podem ser tomadas a respeito da maneira mais correta de interagir com essas plantas, desde um simples monitoramento de infestação e distribuição dessas espécies dentro de um sistema aquático, até uma prática de controle (Martins et al., 2003).

O monitoramento periódico das macrófitas aquáticas permite avaliar a evolução das comunidades e determinar o potencial de danos associados a essas populações. A identificação de focos iniciais de plantas de alto risco para a produção de energia elétrica é bastante importante na tomada de decisões quanto à erradicação ou não desses focos (Carvalho et al., 2005).

Assim, o objetivo deste trabalho foi identificar as plantas aquáticas e os níveis de infestação de cada espécie no reservatório da Usina Hidrelétrica Engenheiro Sérgio Motta (UHE Porto Primavera), visando determinar o potencial de dano dessas populações ao ambiente e à utilização da água para suas várias finalidades, especialmente a geração de energia elétrica.

\section{MATERIAL E MÉTODOS}

A Usina Hidrelétrica Engenheiro Sérgio Motta (UHE Porto Primavera) localiza-se no rio Paraná, a $28 \mathrm{~km}$ a montante da confluência com o rio Paranapanema, e seu reservatório possui uma área de $2.250 \mathrm{~km}^{2}$ (CESP, 2008).

A atividade foi realizada conjuntamente pela CESP-Diretoria de Meio Ambiente, Faculdade de Ciências Agrárias e Veterinárias e pela Faculdade de Ciências Agronômicas, sendo ambas pertencente à UNESP.

O levantamento e a identificação das plantas aquáticas foram realizados no mês de maio de 1998, quando foi concluída a primeira etapa do enchimento do reservatório, na cota de 253,00 m; a segunda etapa foi concluída em março de 2001, com cota de 257,00 m (CESP, 2008). 
O monitoramento foi iniciado através de um voo de aeronave, que compreendeu o rio Paraná e tributários, entre as barragens das usinas hidrelétricas de Eng. Souza Dias (Jupiá) e Porto Primavera, quando foram identificadas as áreas com infestações de plantas aquáticas emersas e imersas no rio/reservatório e lagoas marginais. As áreas com concentrações de plantas aquáticas (26 no total) foram fotografadas e identificadas com auxílio de um GPS portátil, modelo Garmin 12, que também forneceu as suas coordenadas (latitude e longitude) (Tabela 1).

Posteriormente, foram realizadas inspeções utilizando-se barcos, os quais percorreram as duas margens do rio Paraná, de Jupiá a Porto Primavera. No total, foram demarcados 70 pontos de amostragem, no período de 8 a 10 de maio de 1998; a cada 20 minutos navegados, fez-se a leitura das coordenadas no localizador geográfico GPS 12 (Tabela 2). Um gancho foi arrastado no fundo do reservatório, nos pontos demarcados, com o objetivo de verificar a presença ou ausência de plantas submersas. Nos pontos em que foram encontradas plantas emersas, procedeu-se à identificação das espécies e à avaliação da área total infestada e da composição da participação de cada espécie (em porcentagem da área total). Nos casos em que não foi possivel identificar a espécie, uma amostra foi coletada e preservada em sacos plásticos com água, para posterior identificação no laboratório.

Além desses pontos, foram definidos outros, de acordo com o interesse, podendo ser: entradas de tributários, infestações encontradas ao longo do rio (entre os pontos a cada 20 minutos) ou pontos definidos durante o voo realizado anteriormente.

Na Tabela 2 são apresentadas as coordenadas dos pontos de amostragem e as avaliações das populações de plantas aquáticas. Quanto à identificação dos pontos de avaliação, definiu-se o seguinte padrão:

- Áreas identificadas durante o sobrevoo: letras A a Z.

- Áreas identificadas durante a vistoria com barcos:

- Margem direita (Estado de São Paulo): números impares, de 1 a 71 ; e
- Margem esquerda (Estado do Mato Grosso do Sul): números pares, de 2 a 72 .

Determinaram-se as frequências absoluta e relativa de cada espécie por bacia hidrográfica e por conceito de infestação, empregando as seguintes fórmulas (Mueller-Dombois \& Ellemberg, 1974; Braun-Blanquet, 1979):

$$
\mathrm{Fa}=\mathrm{p} / \mathrm{P} .100
$$

em que $\mathrm{Fa}=$ frequência absoluta; $\mathrm{p}=$ número de pontos onde apareceu determinada espécie; e $\mathrm{P}=$ número total de pontos amostrados por bacia hidrográfica.

$$
\mathrm{Fr}=\mathrm{Fae} / \Sigma \mathrm{Fa} .100
$$

em que $\mathrm{Fr}=$ frequência relativa; Fae = frequência absoluta de determinada espécie; e $\Sigma \mathrm{Fa}=$ somatório das frequências absolutas.

Tabela 1 - Coordenadas dos pontos com populações de plantas aquáticas identificadas durante o sobrevoo. Monitoramento, realizado em maio/1998, da área compreendida entre a jusante do reservatório de Jupiá e a montante do reservatório de

\begin{tabular}{|c|c|c|}
\hline Ponto & Latitude (S) & Longitude (W) \\
\hline A & $21^{\circ} 55^{\prime} 24,0^{\prime \prime}$ & $52^{\circ} 15^{\prime} 42,0^{\prime \prime}$ \\
\hline $\mathrm{B}$ & $22^{\circ} 11^{\prime} 10,0^{\prime \prime}$ & $52^{\circ} 26^{\prime} 40,0^{\prime \prime}$ \\
\hline $\mathrm{C}$ & $22^{\circ} 15^{\prime} 06,0^{\prime \prime}$ & $52^{\circ} 36^{\prime} 18,0^{\prime \prime}$ \\
\hline $\mathrm{D}$ & $22^{\circ} 17^{\prime} 27,0^{\prime \prime}$ & $52^{\circ} 39^{\prime} 48,0^{\prime \prime}$ \\
\hline $\mathrm{E}$ & $22^{\circ} 19^{\prime} 48,0^{\prime \prime}$ & $52^{\circ} 43^{\prime} 41,0^{\prime \prime}$ \\
\hline $\mathrm{F}$ & $22^{\circ} 19^{\prime} 42,6^{\prime \prime}$ & $52^{\circ} 43^{\prime} 48,6^{\prime \prime}$ \\
\hline $\mathrm{G}$ & $22^{\circ} 17^{\prime} 25,6^{\prime \prime}$ & $52^{\circ} 48^{\prime} 38,0^{\prime \prime}$ \\
\hline $\mathrm{H}$ & $22^{\circ} 15^{\prime} 38,8^{\prime \prime}$ & $52^{\circ} 37^{\prime} 33,3^{\prime \prime}$ \\
\hline I & $22^{\circ} 14 ' 25,0^{\prime \prime}$ & $52^{\circ} 34^{\prime} 54,2^{\prime \prime}$ \\
\hline $\mathrm{J}$ & $22^{\circ} 13^{\prime} 27,5^{\prime \prime}$ & $52^{\circ} 32^{\prime} 12,1^{\prime \prime}$ \\
\hline $\mathrm{K}$ & $22^{\circ} 11^{\prime} 14,6^{\prime \prime}$ & $52^{\circ} 28^{\prime} 55,6^{\prime \prime}$ \\
\hline $\mathrm{L}$ & $22^{\circ} 10^{\prime} 26,6^{\prime \prime}$ & $52^{\circ} 27^{\prime} 28,3^{\prime \prime}$ \\
\hline $\mathrm{M}$ & $22^{\circ} 03^{\prime} 52,7^{\prime \prime}$ & $52^{\circ} 22^{\prime} 18,4^{\prime \prime}$ \\
\hline $\mathrm{N}$ & $22^{\circ} 05^{\prime} 20,4^{\prime \prime}$ & $52^{\circ} 23^{\prime} 18,7^{\prime \prime}$ \\
\hline $\mathrm{O}$ & $22^{\circ} 02^{\prime} 43,1^{\prime \prime}$ & $52^{\circ} 21^{\prime} 43,7^{\prime \prime}$ \\
\hline $\mathrm{P}$ & $21^{\circ} 44^{\prime} 27,5^{\prime \prime}$ & $52^{\circ} 10^{\prime} 13,9^{\prime \prime}$ \\
\hline Q & $21^{\circ} 40^{\prime} 07,4^{\prime \prime}$ & $52^{\circ} 11^{\prime} 30,9^{\prime \prime}$ \\
\hline $\mathrm{R}$ & $21^{\circ} 40^{\prime} 09,0^{\prime \prime}$ & $52^{\circ} 02^{\prime} 45,0^{\prime \prime}$ \\
\hline $\mathrm{S}$ & $21^{\circ} 36^{\prime} 32,5^{\prime \prime}$ & $52^{\circ} 04^{\prime} 47,1^{\prime \prime}$ \\
\hline $\mathrm{T}$ & $21^{\circ} 33^{\prime} 42,1^{\prime \prime}$ & $52^{\circ} 05^{\prime} 19,0^{\prime \prime}$ \\
\hline $\mathrm{U}$ & $21^{\circ} 31^{\prime} 03,4^{\prime \prime}$ & $51^{\circ} 57^{\prime} 39,7^{\prime \prime}$ \\
\hline $\mathrm{V}$ & $21^{\circ} 27^{\prime} 14,2^{\prime \prime}$ & $51^{\circ} 57^{\prime} 29,4^{\prime \prime}$ \\
\hline W & $21^{\circ} 23^{\prime} 36,2^{\prime \prime}$ & $51^{\circ} 55^{\prime} 48,1^{\prime \prime}$ \\
\hline $\mathrm{X}$ & $21^{\circ} 19^{\prime} 23,4^{\prime \prime}$ & $51^{\circ} 52^{\prime} 48,7^{\prime \prime}$ \\
\hline $\mathrm{Y}$ & $20^{\circ} 55^{\prime} 18,2^{\prime \prime}$ & $51^{\circ} 40^{\prime} 09,4^{\prime \prime}$ \\
\hline $\mathrm{Z}$ & $20^{\circ} 54^{\prime} 16,4^{\prime \prime}$ & $51^{\circ} 39^{\prime} 34,7^{\prime \prime}$ \\
\hline
\end{tabular}
Porto Primavera 
Tabela 2 - Diferentes espécies de plantas aquáticas encontradas no rio Paraná (trecho entre a jusante do reservatório de Jupiá e a montante de Porto Primavera). Monitoramento realizado em maio/1998

\begin{tabular}{|l|l|l|c|}
\hline \multicolumn{1}{|c|}{ Nome Vulgar } & \multicolumn{1}{c|}{ Nome Científico } & \multicolumn{1}{c|}{ Família } & Grupo \\
\hline Alface-d'água & Pistia stratiotes L. & Araceae & F \\
\hline Erva-de-jacaré & Alternanthera philoxeroides (Mart.) Griseb. & Amaranthaceae & E \\
\hline Capim-de-uma-só-cabeça & Cyperus brevifolius (Rottb.) Hassk & Cyperaceae & E \\
\hline Junco-manso & Eleocharis sellowiana Kunth & Cyperaceae & E \\
\hline Elódea densa & Egeria densa Planch. & Hydrocharitaceae & S \\
\hline Elódea najas & Egeria najas Planch. & Hydrocharitaceae & S \\
\hline Lírio-aquático & Nymphaea ampla DC. & Nymphaceae & EF \\
\hline Capim-ferro & Paspalum conspersum Schrad. & Poaceae & E \\
\hline Canarana-rasteira & Paspalum repens Berg. & Poaceae & E \\
\hline Capituva & Echinochloa polystachya (H.B.K.) Hitch. & Poaceae & E \\
\hline Erva-de-bicho & Polygonum hidropiperoides Mich. & Polygonaceae & E \\
\hline Erva-de-bicho & Polygonum lapathifolium L. & Polygonaceae & E \\
\hline Aguapé & Eichhornia crassipes (Mart.) Solms & Pontederiaceae & F \\
\hline Aguapé-de-cordão & Eichhornia azurea (Sw.) Kunth & Pontederiaceae & F \\
\hline Orelha-de-veado & Pontederia cordata L. & Pontederiaceae & E \\
\hline Salvínia & Salvinia auriculata Aubl. & Salviniaceae & F \\
\hline Salvínia & Salvinia rotundifolia Willd. & Salviniaceae & F \\
\hline Taboa & Typha angustifolia L. & Typhaceae & E \\
\hline
\end{tabular}

Grupo: E - emersa; EF - emersa com folhas flutuantes; F - flutuantes; S - submersa.

As espécies foram classificadas nos seguintes grupos (Esteves, 1998; Tanaka et al., 2002b): grupo 1 - emersas: são enraizadas e apresentam maior parte do caule e das folhas acima da superfície da água, desenvolvendose em locais rasos ou ao longo da margem; grupo 2 - emersas com folhas flutuantes: são enraizadas e têm folhas flutuantes, conectadas por caules submersos; grupo 3 - submersas: possuem a maior parte das folhas e do caule abaixo da superficie da água, muitas vezes com flores acima desta superficie, e podem ser enraizadas ou livres; e grupo 4 flutuantes: suas raízes ficam livres na água e apresentam folhas e flores acima da superficie.

\section{RESULTADOS E DISCUSSÃO}

\section{Observação aérea}

Durante o sobrevoo realizado entre a represa de Jupiá e a de Porto Primavera não foi observada quase nenhuma formação de plantas aquáticas (agrupamentos ou moitas) no leito principal do rio Paraná, porém, quando isso ocorreu, eram apenas pequenos agrupamentos de plantas emersas, não sendo visível nenhuma formação de plantas imersas. O aparecimento destas plantas (emersas), no leito do rio, ocorreu mais próximo à barragem de Porto Primavera, em quantidades mais visiveis, junto a "paliteiros" (originários tanto da mata existente nas ilhas como da mata ciliar), sendo esta uma área já alagada, onde não se diferenciava o leito original do rio e a sua antiga margem direita (Mato Grosso do Sul).

Entretanto, nas entradas de tributários e lagoas marginais (principalmente) ou adjacentes ao rio Paraná, tanto na margem esquerda (São Paulo) como na direita (Mato Grosso do Sul), foi observada grande quantidade de plantas aquáticas, sobretudo emersas.

\section{Observação através de barcos}

Na Tabela 2 encontra-se o número de espécies identificadas no reservatório, com os respectivos nomes científicos, família, nomes vulgares e grupo. Foram identificadas 18 espécies, distribuídas em dez famílias de plantas 
aquáticas, vegetando no reservatório. Não houve espécies dominantes com mais de 50\% de ocupação média da área vegetada, porém algumas mostraram-se codominantes.

As espécies encontradas no reservatório foram: Eichhornia crassipes, Eichhornia azurea, Pistia stratiotes, Paspalum repens, Cyperus brevifolius, Paspalum conspersum, Echinochloa polystachya, Egeria densa, Egeria najas, Polygonum hidropiperoides, Polygonum lapathifolium, Alternanthera philoxeroides, Eleocharis sellowiana, Nymphaea ampla, Pontederia cordata, Salvinia auriculata, Salvinia rotundifolia e Typha angustifolia.

Constatou-se a quase inexistência de plantas aquáticas dentro do leito principal do rio Paraná, sendo a maioria das espécies identificadas pertencentes ao grupo das emersas, as quais são caracterizadas como plantas marginais de lugares rasos. Esse tipo de planta tende a desenvolver-se para o interior dos reservatórios, reduzindo a sua capacidade de acúmulo de água. De outra forma, a ondulação da água pode, ainda, promover o deslocamento de grandes blocos de plantas que se movimentam no interior do reservatório, constituindo um risco para a navegação.
O trecho do rio Paraná entre a jusante do reservatório de Jupiá e o município de Presidente Epitácio mostrou-se quase livre de vegetação aquática. No segundo trecho, entre o município de Presidente Epitácio e a barragem de Porto Primavera, foram encontradas grandes formações de plantas emersas apenas próximo à barragem e junto a "paliteiros" de árvores (área já alagada), como observado durante o sobrevoo.

De modo geral, o trecho monitorado apresentou-se livre de comunidades de plantas aquáticas, sendo a área infestada de 12,7 ha muito pequena em comparação à extensão avaliada - 72 pontos de amostragem em cerca de $250 \mathrm{~km}$ ao longo do rio Paraná (Tabela 3).

Em termos de ocorrência na área em estudo (frequência de constatação nas amostragens) (Tabela 4), as principais plantas aquáticas, em ordem decrescente, foram: E. azurea $(36,11 \%)$, E. crassipes $(16,67 \%)$, $P$. stratiotes $(13,89 \%), S$. auriculata $(13,89 \%)$, C. brevifolius $(11,11 \%)$ e $P$. lapathifolium $(6,94 \%)$. Em área de ocorrência $\left(\mathrm{m}^{2}\right)$, as principais plantas foram: E. azurea $(48,56 \%)$, E. crassipes $(40,84 \%)$, P. lapathifolium $(8,51 \%)$, $P$. stratiotes $(0,80 \%), S$. auriculata $(0,73 \%)$ e S. rotundifolia $(0,55 \%)$.

Tabela 3 - Número de pontos (presença de plantas) e área ocupada por diferentes espécies de plantas aquáticas encontradas no rio Paraná (trecho entre a jusante do reservatório de Jupiá e a montante de Porto Primavera). Monitoramento realizado em maio/1998

\begin{tabular}{|c|c|c|c|c|c|c|c|c|c|c|c|c|c|c|c|c|c|c|c|c|}
\hline \multirow{2}{*}{ Ponto } & Latitude & Longitude & \multicolumn{18}{|c|}{ Área infestada pelas plantas aquáticas $\left(\mathrm{m}^{2}\right)$} \\
\hline & (S) & (W) & $\mathrm{Ea}$ & $\mathrm{Ec}$ & Ps & $\mathrm{Sa}$ & $\mathrm{Cb}$ & $\mathrm{Pl}$ & $\mathrm{Ph}$ & Po & $\operatorname{Pr}$ & Ep & $\mathrm{Ta}$ & Ap & Es & Ed & En & $\mathrm{Na}$ & Pc & $\mathrm{Sr}$ \\
\hline 1 & $20^{\circ} 53^{\prime} 56,9^{\prime \prime}$ & $51^{\circ} 36^{\prime} 47,8^{\prime \prime}$ & - & - & - & - & - & - & - & - & - & - & - & - & - & - & - & - & - & - \\
\hline 2 & $20^{\circ} 48^{\prime} 08,4^{\prime \prime}$ & $51^{\circ} 37^{\prime} 54,6^{\prime \prime}$ & - & - & - & - & - & - & - & - & - & - & - & - & - & - & - & - & - & - \\
\hline 3 & $20^{\circ} 53^{\prime} 57,4^{\prime \prime}$ & $51^{\circ} 37^{\prime} 03,1^{\prime \prime}$ & - & - & - & - & - & - & - & - & - & - & - & - & - & - & - & - & - & - \\
\hline 4 & $20^{\circ} 53^{\prime} 28,3^{\prime \prime}$ & $51^{\circ} 38^{\prime} 24,2^{\prime \prime}$ & - & - & - & - & - & - & - & - & - & - & - & - & - & - & - & - & - & - \\
\hline 5 & $20^{\circ} 58^{\prime} 13,9^{\prime \prime}$ & $51^{\circ} 46^{\prime} 05,9^{\prime \prime}$ & - & - & - & - & - & - & - & - & - & - & - & - & - & - & - & - & - & - \\
\hline 6 & $20^{\circ} 58^{\prime} 06,8^{\prime \prime}$ & $51^{\circ} 46^{\prime} 12,4^{\prime \prime}$ & - & - & - & - & - & - & - & - & - & - & - & - & - & ND & ND & - & - & - \\
\hline 7 & $20^{\circ} 58^{\prime} 00,7^{\prime \prime}$ & $51^{\circ} 40^{\prime} 13,5^{\prime \prime}$ & - & - & - & - & - & - & - & - & - & - & - & - & - & - & - & - & - & - \\
\hline 8 & $21^{\circ} 03^{\prime} 38,8^{\prime \prime}$ & $51^{\circ} 46^{\prime} 41,9^{\prime \prime}$ & - & - & - & - & - & - & - & - & - & - & - & - & - & - & - & - & - & - \\
\hline 9 & $21^{\circ} 00^{\prime} 27,7^{\prime \prime}$ & $51^{\circ} 44^{\prime} 23,3^{\prime \prime}$ & - & - & - & - & - & - & - & - & - & - & - & - & - & - & - & - & - & - \\
\hline 10 & $21^{\circ} 07^{\prime} 37,5^{\prime \prime}$ & $51^{\circ} 51^{\prime} 33,3^{\prime \prime}$ & - & - & - & - & - & - & - & - & - & - & - & - & - & - & - & - & - & - \\
\hline 11 & $21^{\circ} 05^{\prime} 12,9^{\prime \prime}$ & $51^{\circ} 46^{\prime} 31,9^{\prime \prime}$ & - & - & ND & ND & - & - & - & - & - & - & - & - & ND & - & - & - & - & - \\
\hline 12 & $21^{\circ} 11^{\prime} 34,1^{\prime \prime}$ & $51^{\circ} 53^{\prime} 07,7^{\prime \prime}$ & ND & - & - & - & - & - & - & - & - & - & - & - & - & - & - & - & - & - \\
\hline 13 & $21^{\circ} 08^{\prime} 11,6^{\prime \prime}$ & $51^{\circ} 50^{\prime} 46,9^{\prime \prime}$ & $\mathrm{ND}$ & - & - & - & - & - & - & - & - & - & - & - & - & - & - & - & - & - \\
\hline 14 & $21^{\circ} 13^{\prime} 28,3^{\prime \prime}$ & $51^{\circ} 52^{\prime} 40,8^{\prime \prime}$ & - & - & - & - & - & - & - & - & - & - & - & - & - & - & - & - & - & - \\
\hline 15 & $21^{\circ} 08^{\prime} 48,6^{\prime \prime}$ & $51^{\circ} 52^{\prime} 36,9^{\prime \prime}$ & - & - & - & - & - & - & - & - & - & - & - & - & - & - & - & - & - & - \\
\hline 16 & $21^{\circ} 18^{\prime} 24,3^{\prime \prime}$ & $51^{\circ} 51^{\prime} 58,4^{\prime \prime}$ & - & - & - & - & - & - & - & - & - & - & - & - & - & - & - & - & - & - \\
\hline 17 & $21^{\circ} 12^{\prime} 06,4^{\prime \prime}$ & $51^{\circ} 51^{\prime 2} 28,1^{\prime \prime}$ & - & - & - & - & - & - & - & - & - & - & - & ND & - & - & - & - & - & - \\
\hline 18 & $21^{\circ} 20^{\prime} 43,4^{\prime \prime}$ & $51^{\circ} 52^{\prime} 04,1^{\prime \prime}$ & - & - & - & - & - & - & - & - & - & - & - & - & - & - & - & - & - & - \\
\hline 19 & $21^{\circ} 15^{\prime} 26,0^{\prime \prime}$ & $51^{\circ} 50^{\prime} 49,3^{\prime \prime}$ & $\mathrm{ND}$ & - & - & - & - & - & - & - & - & - & - & - & - & - & - & - & - & - \\
\hline 20 & $21^{\circ} 24^{\prime} 04,4^{\prime \prime}$ & $51^{\circ} 55^{\prime} 11,7^{\prime \prime}$ & $\mathrm{ND}$ & - & - & - & - & - & - & - & - & - & - & - & - & - & - & - & - & - \\
\hline 21 & $21^{\circ} 20^{\prime} 27,8^{\prime \prime}$ & $51^{\circ} 51^{\prime} 19,2^{\prime \prime}$ & - & - & - & - & - & - & - & - & - & - & - & - & - & - & - & - & - & - \\
\hline
\end{tabular}

Continua... 
Tabela 3 , Cont

\begin{tabular}{|c|c|c|c|c|c|c|c|c|c|c|c|c|c|c|c|c|c|c|c|c|}
\hline \multirow{2}{*}{ Ponto } & \multirow{2}{*}{$\begin{array}{c}\text { Latitude } \\
\text { (S) }\end{array}$} & \multirow{2}{*}{\begin{tabular}{|c|} 
Longitude \\
(W)
\end{tabular}} & \multicolumn{18}{|c|}{ Área infestada pelas plantas aquáticas $\left(\mathrm{m}^{2}\right)$} \\
\hline & & & $\mathrm{Ea}$ & $\mathrm{Ec}$ & Ps & $\mathrm{Sa}$ & $\mathrm{Cb}$ & Pl & $\mathrm{Ph}$ & Po & $\operatorname{Pr}$ & Ep & $\mathrm{Ta}$ & $\mathrm{Ap}$ & Es & $\mathrm{Ed}$ & En & $\mathrm{Na}$ & $\mathrm{Pc}$ & $\mathrm{Sr}$ \\
\hline 22 & $21^{\circ} 24^{\prime} 32,6^{\prime \prime}$ & $51^{\circ} 55^{\prime} 19,9^{\prime \prime}$ & - & - & - & - & - & - & - & - & - & - & - & - & - & - & - & - & - & - \\
\hline 23 & $21^{\circ} 24^{\prime} 28,2^{\prime \prime}$ & $51^{\circ} 54^{\prime} 43,6^{\prime \prime}$ & - & - & - & - & - & - & - & - & - & - & - & - & - & - & - & - & - & - \\
\hline 24 & $21^{\circ} 26^{\prime} 57,3^{\prime \prime}$ & $51^{\circ} 55^{\prime} 50,8^{\prime \prime}$ & 3 & - & - & - & - & - & - & - & - & - & - & - & - & - & - & - & - & - \\
\hline 25 & $21^{\circ} 27^{\prime} 59,9^{\prime \prime}$ & $51^{\circ} 55^{\prime} 35,8^{\prime \prime}$ & - & - & - & - & - & - & - & - & - & - & - & - & - & - & - & - & - & - \\
\hline 26 & $21^{\circ} 27^{\prime} 40,5^{\prime \prime}$ & $51^{\circ} 56^{\prime} 20,6^{\prime \prime}$ & - & - & - & - & - & - & - & - & - & - & - & - & - & - & - & - & - & - \\
\hline 27 & $21^{\circ} 30^{\prime} 03,6^{\prime \prime}$ & $51^{\circ} 57^{\prime} 05,6^{\prime \prime}$ & - & - & - & - & - & - & - & - & - & - & - & - & - & - & - & - & - & - \\
\hline 28 & $21^{\circ} 30^{\prime} 07,0^{\prime \prime}$ & $51^{\circ} 58^{\prime} 35,1^{\prime \prime}$ & - & - & - & - & - & - & - & - & - & - & - & - & - & - & - & - & - & - \\
\hline 29 & $21^{\circ} 30^{\prime} 57,8^{\prime \prime}$ & $51^{\circ} 57^{\prime} 45,8^{\prime \prime}$ & 300 & - & - & - & - & - & - & - & - & 200 & - & - & - & - & - & - & - & - \\
\hline 30 & $21^{\circ} 31^{\prime} 14,9^{\prime \prime}$ & $52^{\circ} 00^{\prime} 13,2^{\prime \prime}$ & - & - & - & ND & - & - & - & ND & - & - & - & - & - & - & - & - & - & - \\
\hline 31 & $21^{\circ} 30^{\prime} 51,1^{\prime \prime}$ & $51^{\circ} 58^{\prime} 52,6^{\prime \prime}$ & - & ND & - & - & - & - & - & - & - & - & - & - & - & - & - & - & - & - \\
\hline 32 & $21^{\circ} 43^{\prime} 07,1^{\prime \prime}$ & $52^{\circ} 10^{\prime} 22,0^{\prime \prime}$ & - & - & - & - & - & - & - & - & - & - & - & - & - & - & - & - & - & - \\
\hline 33 & $21^{\circ} 31^{\prime} 33,5^{\prime \prime}$ & $52^{\circ} 04^{\prime} 26,1^{\prime \prime}$ & - & - & - & - & - & - & - & - & - & - & - & - & - & - & - & - & - & - \\
\hline 34 & $21^{\circ} 44^{\prime} 11,0^{\prime \prime}$ & $52^{\circ} 10^{\prime} 03,2^{\prime \prime}$ & - & - & - & - & - & ND & ND & - & ND & - & - & - & - & - & - & - & - & - \\
\hline 35 & $21^{\circ} 34^{\prime} 36,2^{\prime \prime}$ & $52^{\circ} 05^{\prime} 21,0^{\prime \prime}$ & - & - & - & - & - & - & - & - & - & - & - & - & - & - & - & - & - & - \\
\hline 36 & $21^{\circ} 44^{\prime} 35,4^{\prime \prime}$ & $52^{\circ} 10^{\prime} 24,3^{\prime \prime}$ & ND & - & - & - & - & - & ND & - & - & - & - & - & - & - & - & - & - & - \\
\hline 37 & $21^{\circ} 38^{\prime} 19,7^{\prime \prime}$ & $52^{\circ} 02^{\prime} 40,2^{\prime \prime}$ & - & - & - & - & - & - & - & - & - & - & - & - & - & - & - & - & - & - \\
\hline 38 & $21^{\circ} 45^{\prime} 13,2^{\prime \prime}$ & $52^{\circ} 10^{\prime} 22,3^{\prime \prime}$ & - & - & - & - & - & - & - & - & - & - & - & - & - & - & - & - & - & - \\
\hline 39 & $21^{\circ} 42^{\prime} 09,0^{\prime \prime}$ & $52^{\circ} 03^{\prime} 23,0^{\prime \prime}$ & ND & - & - & - & - & - & ND & - & ND & - & - & - & - & - & - & - & - & - \\
\hline 40 & $21^{\circ} 45^{\prime} 50,5^{\prime \prime}$ & $52^{\circ} 09^{\prime} 54,9^{\prime \prime}$ & - & - & - & - & - & - & - & - & - & - & - & - & - & - & - & - & - & - \\
\hline 41 & $21^{\circ} 44^{\prime} 31,6^{\prime \prime}$ & $52^{\circ} 05^{\prime} 00,7^{\prime \prime}$ & ND & - & - & ND & - & - & ND & - & - & - & - & - & - & - & - & - & ND & - \\
\hline 42 & $21^{\circ} 44^{\prime} 52,5^{\prime \prime}$ & $52^{\circ} 07^{\prime} 58,9^{\prime \prime}$ & - & - & - & - & - & - & - & - & - & - & - & - & - & - & - & - & - & - \\
\hline 43 & $21^{\circ} 50^{\prime} 00,5^{\prime \prime}$ & $52^{\circ} 10^{\prime} 41,9^{\prime \prime}$ & - & - & - & - & - & - & - & - & - & 200 & - & - & - & - & - & - & - & - \\
\hline 44 & $21^{\circ} 42^{\prime} 51,5^{\prime \prime}$ & $52^{\circ} 05^{\prime} 02,7^{\prime \prime}$ & - & - & - & - & - & - & - & - & - & - & - & - & - & - & - & - & - & - \\
\hline 45 & $21^{\circ} 52^{\prime} 03,1^{\prime \prime}$ & $52^{\circ} 12^{\prime} 21,4^{\prime \prime}$ & - & - & - & - & - & - & - & - & - & - & - & - & - & - & - & - & - & - \\
\hline 46 & $21^{\circ} 38^{\prime} 23,5^{\prime \prime}$ & $52^{\circ} 03^{\prime} 20,8^{\prime \prime}$ & $\mathrm{ND}$ & - & - & - & ND & - & - & - & - & - & - & - & - & - & - & - & - & - \\
\hline 47 & $21^{\circ} 52^{\prime} 55,0^{\prime \prime}$ & $52^{\circ} 13^{\prime} 33,4^{\prime \prime}$ & - & - & - & - & - & - & - & 3 & - & - & - & - & - & - & - & - & - & - \\
\hline 48 & $21^{\circ} 34^{\prime} 18,8^{\prime \prime}$ & $52^{\circ} 06^{\prime} 38,0^{\prime \prime}$ & ND & ND & ND & & ND & - & - & - & - & - & - & - & - & - & - & - & - & - \\
\hline 49 & $21^{\circ} 55^{\prime} 12,9^{\prime \prime}$ & $52^{\circ} 15^{\prime} 42,9^{\prime \prime}$ & ND & - & - & - & - & - & - & - & - & - & - & - & - & - & - & - & - & - \\
\hline 50 & $21^{\circ} 32^{\prime} 01,4^{\prime \prime}$ & $52^{\circ} 05^{\prime} 55,6^{\prime \prime}$ & - & - & - & - & - & - & - & - & - & - & - & - & - & - & - & - & - & - \\
\hline 51 & $21^{\circ} 59^{\prime} 42,9^{\prime \prime}$ & $52^{\circ} 18^{\prime} 44,2^{\prime \prime}$ & 4 & - & - & - & - & - & - & - & - & - & - & - & - & - & - & - & - & - \\
\hline 52 & $21^{\circ} 30^{\prime} 36,5^{\prime \prime}$ & $52^{\circ} 00^{\prime} 31,7^{\prime \prime}$ & - & - & - & - & - & - & - & - & - & - & - & - & - & - & - & - & - & - \\
\hline 53 & $22^{\circ} 01^{\prime} 56,0^{\prime \prime}$ & $52^{\circ} 19^{\prime} 40,1^{\prime \prime}$ & 540 & 18 & 30 & 12 & - & - & - & - & - & - & - & - & - & - & - & - & - & - \\
\hline 54 & $21^{\circ} 52^{\prime} 27,7^{\prime \prime}$ & $52^{\circ} 14^{\prime} 21,2^{\prime \prime}$ & ND & - & - & - & ND & - & - & - & - & - & - & - & - & - & - & - & - & - \\
\hline 55 & $22^{\circ} 04^{\prime} 08,3^{\prime \prime}$ & $52^{\circ} 20^{\prime} 15,5^{\prime \prime}$ & ND & ND & ND & ND & - & ND & - & - & - & - & - & - & - & - & - & - & - & - \\
\hline 56 & $21^{\circ} 54^{\prime} 08,5^{\prime \prime}$ & $52^{\circ} 15^{\prime} 26,2^{\prime \prime}$ & 320 & - & 12 & 60 & 8 & - & - & - & - & - & - & - & - & - & - & - & - & - \\
\hline 57 & $22^{\circ} 11^{\prime} 03,3^{\prime \prime}$ & $52^{\circ} 26^{\prime} 37,0^{\prime \prime}$ & 120 & 2 & 15 & ND & ND & 14 & - & - & - & - & - & - & - & - & - & - & - & - \\
\hline 58 & $21^{\circ} 54^{\prime} 49,9^{\prime \prime}$ & $52^{\circ} 16^{\prime} 27,9^{\prime \prime}$ & 10 & - & - & - & - & - & - & - & - & - & - & - & - & - & - & 2 & - & - \\
\hline 59 & $22^{\circ} 16^{\prime} 18,0^{\prime \prime}$ & $52^{\circ} 34^{\prime} 58,2^{\prime \prime}$ & - & - & - & - & - & - & - & - & - & - & - & - & - & - & - & - & - & - \\
\hline 60 & $21^{\circ} 58^{\prime} 53,6^{\prime \prime}$ & $52^{\circ} 19^{\prime} 38,8^{\prime \prime}$ & - & - & - & - & - & - & - & - & - & - & - & - & - & - & - & - & - & - \\
\hline 61 & $21^{\circ} 18^{\prime} 22,5^{\prime \prime}$ & $52^{\circ} 38^{\prime} 32,4^{\prime \prime}$ & 600 & 250 & 250 & 150 & - & 3750 & - & - & - & - & - & - & - & - & - & - & - & - \\
\hline 62 & $22^{\circ} 06^{\prime} 31,4^{\prime \prime}$ & $52^{\circ} 23^{\prime} 45,5^{\prime \prime}$ & ND & - & - & - & - & - & - & ND & - & - & - & - & - & - & - & - & - & - \\
\hline 63 & $22^{\circ} 21^{\prime} 16,9^{\prime \prime}$ & $52^{\circ} 43^{\prime} 33,7^{\prime \prime}$ & 40 & 8 & 2 & - & 1 & - & - & - & - & - & - & - & - & - & - & - & - & - \\
\hline 64 & $22^{\circ} 11^{\prime} 37,8^{\prime \prime}$ & $52^{\circ} 28^{\prime} 51,3^{\prime \prime}$ & - & - & - & - & - & - & - & - & - & - & - & - & - & - & - & - & - & - \\
\hline 65 & $22^{\circ} 22^{\prime} 55,8^{\prime \prime}$ & $52^{\circ} 46^{\prime} 45,1^{\prime \prime}$ & - & 50000 & - & - & ND & - & - & - & - & - & - & - & - & - & - & - & - & - \\
\hline 66 & $22^{\circ} 16^{\prime} 07,0^{\prime \prime}$ & $52^{\circ} 37^{\prime} 17,6^{\prime \prime}$ & ND & ND & ND & ND & ND & - & - & - & - & - & ND & - & - & - & - & - & - & - \\
\hline 67 & $22^{\circ} 25^{\prime} 57,7^{\prime \prime}$ & $52^{\circ} 50^{\prime} 06,7^{\prime \prime}$ & - & ND & - & - & - & - & - & - & - & - & - & - & - & - & - & - & - & - \\
\hline 68 & $22^{\circ} 19^{\prime} 32,9^{\prime \prime}$ & $52^{\circ} 43^{\prime} 34,8^{\prime \prime}$ & 59500 & 1400 & 700 & 700 & - & 7000 & - & - & - & - & - & - & - & - & - & - & - & 700 \\
\hline 69 & $22^{\circ} 27^{\prime} 47,4^{\prime \prime}$ & $52^{\circ} 53^{\prime} 02,8^{\prime \prime}$ & ND & ND & - & - & - & - & - & - & - & - & ND & - & - & - & - & - & - & - \\
\hline 70 & $22^{\circ} 19^{\prime} 50,0^{\prime \prime}$ & $52^{\circ} 43^{\prime} 39,9^{\prime \prime}$ & - & - & - & - & - & - & - & - & - & - & - & - & - & - & - & - & - & - \\
\hline 71 & $22^{\circ} 28^{\prime} 45,8^{\prime \prime}$ & $52^{\circ} 55^{\prime} 23,8^{\prime \prime}$ & - & - & - & - & - & - & - & - & - & - & - & - & - & - & - & - & - & - \\
\hline 72 & $22^{\circ} 23^{\prime} 58,8^{\prime \prime}$ & $52^{\circ} 50^{\prime} 00,5^{\prime \prime}$ & ND & - & - & - & - & - & - & - & - & - & - & - & - & - & - & - & - & - \\
\hline
\end{tabular}

(ND) - área não dimensionada (presença apenas de poucas plantas).

$(-)$ - sem plantas 
A espécie E. azurea apresentou a maior frequência absoluta e a maior área de infestação nesse reservatório. Segundo Martins et al. (2003), essa também foi a mais importante espécie daninha encontrada nos reservatórios da Light, RJ. Em um outro estudo, Tanaka et al. (2002), ao realizarem um levantamento de plantas aquáticas nos reservatórios da bacia do Rio Paraná em 1999, identificaram 17 espécies e verificaram que as espécies flutuantes (E. crassipes, P. stratiotes, Salvinia molesta e Salvinia minima) e as emersas (Cyperus sp. e E. azurea) foram encontradas com mais frequência no reservatório de Porto Primavera, demonstrando que $E$. azurea continua sendo importante no reservatório.

Das 18 espécies encontradas no primeiro levantamento florístico realizado no reservatório de Porto Primavera em maio de 1998, foram observadas apenas cinco no levantamento realizado em 1999 (Tanaka et al., 2002), sendo elas: E. crassipes, E. azurea, P. stratiotes, $P$. repens e $P$. hidropiperoides. Esse fato evidencia que, após o enchimento do lago, as condições do ambiente e/ou a interferência entre as espécies existentes no reservatório foram modificadas com o tempo.

Em um levantamento realizado em 2002 na Bacia do Rio Paraná, Martins et al. (2008) identificaram 28 espécies, entre as quais as cinco mais frequentes foram: $S$. auriculata, E. crassipes, Cyperus sp., P. stratiotes e Typha latifolia, encontradas em 97,4; 79,5; 51,3; 46,2; e $46,2 \%$ dos pontos amostrados, respectivamente. Do total das espécies identificadas, 50,0 e $53,57 \%$ foram encontradas nos reservatórios de Porto Primavera e de Jupiá, respectivamente, destacando-se o reservatório de Ilha Solteira, com $85,7 \%$ das espécies.

Das 18 espécies encontradas no primeiro levantamento florístico realizado no reservatório de Porto Primavera em maio de 1998, observaram-se apenas nove no levantamento realizado em 2002 (Martins et al., 2008). Ressalta-se que $P$. repens, $P$. conspersum, E. densa, P. hidropiperoides, A. philoxeroides, E. sellowiana, N. ampla, P. cordata e $S$. rotundifolia não foram encontradas no segundo levantamento (2002). Contudo, cinco novas espécies foram identificadas, destacando-se: Echinochloa sp., Ipomoea sp., Ludwigia elegans, Ludwigia sericea e Mimosa glabra.

No levantamento realizado em 2002 (Martins et al., 2008), apenas foram encontradas cinco espécies que haviam sido observadas no levantamento de 1999 (Tanaka et al., 2002), destacando-se Cyperus sp., E. crassipes, E. azurea, P. stratiotes e T. latifolia.

As espécies do grupo das plantas submersas E. najas e E. densa apresentaram frequência absoluta e relativa na ordem de 1,43 e $1,15 \%$, respectivamente. É importante destacar que essas espécies são consideradas plantas daninhas aquáticas que causam sérios inconvenientes à geração de energia no reservatório de Jupiá, localizado a montante do reservatório de Porto Primavera (Marcondes et al., 2003).

Todos os tributários visitados, tanto do lado direito como do lado esquerdo do rio Paraná, apresentavam grande quantidade de plantas aquáticas emersas, não sendo verificadas plantas imersas. Também, todas as lagoas adjacentes ao rio Paraná, em ambas as margens, apresentavam formações de plantas aquáticas, sendo principalmente de emersas; essa constatação foi facilitada pela existência de canais que ligavam as lagoas ao leito do rio Paraná.

Em alguns locais, onde as lagoas eram interiores, sem ligação direta com o leito do rio, os barcos foram deixados e caminhou-se em direção a esses pontos, que ficavam distantes das margens - estes locais foram identificados anteriormente por via aérea. Contudo, a mata ciliar e as áreas alagadas ou pantanosas dificultaram o acesso direto a esses locais, impedindo uma melhor visualização das lagoas marginais, o que permitiu apenas verificar a existência de plantas aquáticas nestas áreas, sem mais pormenores. Com o enchimento do lago, o acesso a esses locais não será dificultado, uma vez que se registraram, via aérea, as coordenadas geográficas desses pontos com GPS. 
Foi evidenciado que os tributários, lagoas marginais do rio Paraná e os reservatórios localizados a montante do reservatório de Porto Primavera podem ser destacados como os maiores fornecedores de plantas aquáticas ao leito principal do reservatório de Porto Primavera. A grande ocorrência de plantas aquáticas nesses ambientes pode estar relacionada, entre outros fatores, à riqueza da água e ao menor fluxo, o que levaria a um rápido crescimento e desenvolvimento dessas plantas, sob condições de baixo estresse.

As informações geradas possibilitam o planejamento de operações de manejo e controle subsequentes, pois, dessa forma, pode-se conhecer o nível de infestação de diferentes espécies, em áreas pontuais do reservatório, auxiliando na evolução de estratégias de controle dessas espécies de plantas aquáticas.

Os resultados obtidos servirão para comparação com as futuras situações após a formação do reservatório, o que permitirá verificar quais espécies poderão causar prejuízos aos usos da água.

\section{LITERATURA CITADA}

BRANCO, S. M. Hidrobiologia aplicada à engenharia sanitária. 3.ed. São Paulo: CETESB, 1986. 616 p.

BRAUN-BLANQUET, J. Fitossociologia: bases para el estudio de las comunidades vegetales. 3.ed. Madrid: Blume, 1979. $820 \mathrm{p}$.

CARVALHO, F. T.; VELINI, E. D.; MARTINS, D. Plantas aquáticas e nível de infestação das espécies presentes no reservatório de Bariri, no rio Tietê. Planta Daninha, v. 23, n. 2, p. 371-374, 2005.

COMPANHIA ENERGÉTICA DE SÃO PAULO - CESP. Disponível em: http://www.cesp.com.br/. 2008.

ESTEVES, F. A. Fundamentos de limnologia. 2.ed. Rio de Janeiro: Interciência, 1998. 602 p.
GALO, M. L. B. T. et al. Uso do sensoriamento remoto orbital no monitoramento da dispersão de macrófitas nos reservatórios do complexo Tietê. Planta Daninha, v. 20, p. 7-20, 2002. (Edição Especial)

MARCONDES, D.A.S. et al. Eficiência de fluridone no controle de plantas aquáticas submersas no reservatório de Jupiá. Planta Daninha, v.21, p.69-78, 2003. (Edição Especial)

MARSHALL, T. R.; LEE, P. F. Mapping aquatic macrophytes through digital image analysis of aerial photographs: an assessment. J. Aquatic Plant Manag., v. 32, n. 1, p. 61-66, 1994.

MARTINS, D. et al. Ocorrência de plantas aquáticas nos reservatórios da Light-RJ. Planta Daninha, v. 21, p. 105-108, 2003. (Edição Especial)

MARTINS, D. et al. Caracterização da comunidade de plantas aquáticas de dezoito reservatórios pertencentes a cinco bacias hidrográficas do estado de São Paulo. Planta Daninha, v. 26, n. 1, p. 17-32, 2008.

MARTYN, R. D. et al. Mapping aquatic weeds with aerial color infrared photography and evaluating their control by grass carp. J. Aquatic Plant Manag., v. 24, n. 1, p. 46-56, 1986.

MUELLER-DOMBOIS, D.; ELLENBERG, H. Aims and methods of vegetation ecology. New York: John Wiley \& Sons, 1974. $547 \mathrm{p}$

NEVES, T.; FOLONI, L. L.; PITELLI, R. A. Controle químico do aguapé (Eichhornia crassipes). Planta Daninha, v 20, p. 89-97, 2002. (Edição especial)

PITELLI, R. A. Macrófitas aquáticas no Brasil, na condição de problemáticas. In: WORKSHOP SOBRE CONTROLE DE PLANTAS AQUÁTICAS, 1998, Brasília. Anais... Brasília: IBAMA, 1998. p. 12-15.

TANAKA, R. H. et al. Ocorrência de plantas aquáticas nos reservatórios da Companhia Energética de São Paulo. Planta Daninha, v. 20, p. 101-111, 2002. (Edição Especial)

VELINI, E. D. Controle mecânico de plantas aquáticas no Brasil. In: WORKSHOP SOBRE CONTROLE DE PLANTAS AQUÁTICAS, 1998, Brasília. Anais... Brasília: IBAMA, 1998. p. 32-35. 